\title{
Virtudes Organizacionales, Performance y Satisfacción Laboral. Diferencias según variables individuales y organizacionales en empleados argentinos
}

\author{
María Laura Lupano Perugini ${ }^{1}$
}

\begin{abstract}
Artículo
Material original autorizado para la publicación en la revista Psicodebate. Facultad de Ciencias Sociales. Universidad de Palermo.

Recibido 16-02-2017 | Aceptado 02-05-2017
\end{abstract}

\section{Resumen}

El presente trabajo se basa en las propuestas de la Psicología Organizacional Positiva (POP) que intenta integrar a la mirada clásica de estudios organizacionales -que se focaliza en analizar condiciones negativas-, una perspectiva que visualice variables positivas que contribuyan a obtener resultados óptimos (Cameron \& Spreitzer, 2012). Se planteó como objetivo analizar si existen diferencias en las virtudes nivel organizacional (Apoyo y respeto, Significado e inspiración, Perdón) según variables individuales (e.g. personal a cargo) y organizacionales (e.g. tamaño y tipo de empresa). Asimismo, se buscó establecer si existe relación entre dichas virtudes y medidas resultado (performance organizacional/ individual y satisfacción laboral). La muestra estuvo compuesta por 569 empleados/as argentinos (288 hombres, 281 mujeres); edad promedio: 36.85 años $(D E=11.7)$; pertenecientes a empresas públicas $(18.1 \%, n=103)$ y privadas $(81.9 \%, n=466)$. La mayoría residía en ciudad de Buenos Aires y alrededores $(96.8 \%, n=545)$. Para la recolección de datos se empleó: Inventario de virtudes organizacionales -IVO- y algunas encuestas diseñadas ad-hoc: Encuesta sociodemográfica, organizacional, de satisfacción laboral, de desempeño organizacional e individual. Mediante diferentes análisis univariados, se determinó que las personas de mayor edad y aquellos que ocupan una posición de liderazgo presentan niveles más altos de Significado e inspiración. Asimismo, en las empresas de gran tamaño y de rubro no estereotípico, se percibieron menores niveles de Apoyo y respeto. Todas

1 Consejo Nacional de Investigaciones Científicas y Técnicas (CONICET). Universidad de Palermo. Argentina. mllupano@hotmail.com 
las virtudes correlacionaron de manera significativa con medidas de performance y satisfacción.

Palabras clave: performance, satisfacción, virtudes organizacionales.

\title{
Organizational Virtues, Performance and Job Satisfaction. Differences according individual and organizational factors in Argentinean employees
}

\begin{abstract}
This study is based on Positive Organizational Scholarship (POS). That perspective tries to integrate classical organizational studies -which only analyze the negative conditions- with a perspective that visualizes positive variables that contribute to obtain optimal results (Cameron \& Spreitzer, 2012). The present study aimed to find differences in organizational virtues (Support and respect, Meaning and inspiration, Forgiveness), according personal (e.g. position) and organizational variables (e.g. size and type of company). Also, it was sought if exist relation between organizational virtues and outcomes measures (organizational / individual performance and job satisfaction). The sample consisted of 569 Argentinean employees (288 men, 281 women); average age: 36.85 years $(S D=11.7)$; belonging to public companies $(18.1 \%, n=103)$ and private companies $(81.9 \%, n=466)$. Most of them lived in Buenos Aires and surrounding areas $(96.8 \%, n=545)$. For data collection was used: Organizational virtues Inventory - IVO- and surveys designed ad-hoc: socio-demographic, organizational, job satisfaction, organizational and individual performance Surveys. Using univariate analyzes, it was concluded that older people and those in a leadership position have higher levels of Meaning and inspiration. Also, in large and non-stereotypical companies, lower levels of Support and respect were perceived. All virtues correlated significantly with measures of performance and satisfaction.
\end{abstract}

Keywords: performance, satisfaction, organizational virtues. 
El siguiente estudio intenta abordar la relación entre virtudes a nivel organizacional con medidas de satisfacción y performance, considerando diferencias de acuerdo con variables individuales y organizacionales. Las virtudes organizacionales son definidas como atributos o prácticas morales (e.g. justicia, respeto, dignidad) a nivel global dentro de una organización y no como la suma de las virtudes individuales de sus miembros. Serían características percibidas en las organizaciones que influyen en el accionar de las personas y contribuyen a que experimenten una vida más plena, tal como lo hacen las fortalezas a nivel individual (Park \& Peterson, 2003; Peterson, 2006). Cameron y Winn (2012) argumentan que debería hablarse de virtuosidad (virtuosness), en lugar de virtudes, ya que destacan que estas refieren a un nivel individual y la expresión virtuosidad aludiría a una expresión colectiva. De acuerdo con Cameron, Bright y Caza (2004), una definición global incluye tanto acciones individuales como actividades colectivas y atributos culturales y sociales. Además, dichas virtudes presentan dos cualidades: la amplificación (se relaciona con el hecho de que la exposición a prácticas virtuosas incrementaría los niveles de emociones positivas, capital social $\mathrm{y}$ conductas prosociales, retroalimentando asimismo a dichas prácticas) y, por otro lado, la cualidad amortiguadora (esta cualidad ayuda a prevenir efectos negativos, producto de situaciones estresantes, promoviendo un sentido de resiliencia, solidaridad y eficacia). Estas cualidades favorecerían la obtención de resultados positivos y buen rendimiento (Bright, Cameron, \& Caza, 2006).

La perspectiva teórica escogida para el análisis de esta temática es la Psicología Organizacional Positiva (POP), cuyos iniciadores son Dutton, Cameron y Quinn, de la Universidad de Michigan, Estados Unidos (Cameron \& Spreitzer, 2012). $\mathrm{Su}$ surgimiento se vincula con el hecho de que la mayor parte de los estudios organizacionales clásicos han puesto el foco en el análisis de las condiciones negativas y sus consecuencias, en tanto que POP intenta integrar a esta mirada una perspectiva positiva que visualice aquellas variables que contribuyen a resultados óptimos y no sólo las que lo impiden (Lupano Perugini, 2014).

Desde la POP se promueve el desarrollo de organizaciones positivas que se comprometan con el desarrollo y la promoción de la salud de una manera comprehensiva, interdisciplinar y multicausal (Salanova, Llorens \& Martinez, 2016). Si bien el término positivo ha estado asociado a una connotación valórica (Fineman, 2006), en los últimos años se lo ha empezado a vincular con factores orientados a lograr un mejor rendimiento en las organizaciones (Dutton \& Glynn, 2007; Quinn, 2015; Siedel, 2016). En el mundo actual es de fundamental importancia realizar investigaciones que aborden factores que puedan contribuir a aumentar los niveles de satisfacción laboral y performance en las organizaciones. La satisfacción laboral es entendida como una actitud que las personas tienen hacia 
su trabajo (Cameron \& Spreitzer, 2012), aunque también ha sido conceptualizada como una reacción afectiva o una evaluación del trabajo (Weiss \& Breif, 2001). Por lo tanto, involucraría tanto componentes cognitivos - evaluación- como afectivos. Si se pretende analizarla se deben tener en cuenta diferentes facetas (e.g. satisfacción con el supervisor, con los compañeros de trabajo, con la remuneración, con las posibilidades de ascender) así como el trabajo en general (Spector, 1997). Por otro lado, la performance laboral se relaciona con el nivel de desempeño que presenta una persona en las tareas necesarias para un rol laboral y también por su contribución al contexto social y psicológico de una organización (Judge, Thorensen, Bono, \& Patton, 2001).

En relación con lo antedicho, Cameron, Mora, Leutscher y Calarco (2011) realizaron algunas investigaciones en las que comprobaron el efecto de prácticas virtuosas a nivel organizacional sobre los niveles de performance. Por ejemplo, una de las investigaciones se realizó con una muestra de 2386 empleados pertenecientes a organizaciones que no son típicamente asociadas con factores relacionados con prácticas positivas y humanitarias (e.g., atención al cliente, marketing, servicios de inversión). Las variables analizadas fueron: prácticas positivas -virtuosidad-, nivel de rotación de empleados, clima organizacional, rendimiento financiero (medida objetiva) y valoración de experto (medida subjetiva) -efectividad y compromiso-. En todos los casos se obtuvieron correlaciones significativas, dando cuenta de que las prácticas positivas pueden ser un buen predictor de estos factores, incluso en este tipo de organizaciones. En los estudios realizados por este equipo se halló, en general, que las virtudes tienen un mayor efecto -sobre las variables de resultado (e.g. performance)- si se las considera en forma agregada que si se las toma en forma individualizada (Cameron \& Winn, 2012).

En Argentina, se están llevando a cabo una serie de trabajos (como el que se presenta) orientados a verificar la mencionada relación entre dichas virtudes y los niveles de rendimiento y satisfacción laboral. Para ello, se ha realizado la adaptación de un instrumento empleado en las investigaciones de los autores citados (Cameron et al., 2011). El instrumento original es el Positive Practices Survey que propone analizar seis virtudes: Dignidad y respeto, Apoyo, Interés, Significado, Inspiración, Perdón. Como resultado del proceso de validación de esta prueba en la Argentina (Lupano Perugini \& Castro Solano, 2017a), se obtuvo una versión final de tres factores que resultan ser una combinación y reducción de las escalas del instrumento original: Apoyo y respeto, Significado e inspiración, y Perdón.

En un trabajo reciente (Lupano Perugini \& Castro Solano, 2017b) se analizó la relación entre las virtudes arriba mencionadas y una serie de características positivas que los empleados de organizaciones argentinas asociaron con su 
lugar de trabajo. Estas características fueron obtenidas en una investigación anterior (Lupano Perugini \& Castro Solano, 2016) y son las siguientes: clima y relaciones interpersonales, comodidad, remuneración, flexibilidad, organización, oportunidades y capacitación, estabilidad, prestigio, resultados, respeto, valores, compromiso/confianza, diversidad. En dicho estudio, además, se halló que las características positivas que se vincularon con altos niveles de rendimiento y satisfacción fueron aquellas que se relacionan con percibirse eficaces (e.g., "resultados") y con sostener valores que contribuyan a la armonía social (e.g., "ser honesto" o "valorar a otros"). En cambio, los bajos niveles se asociaron a atributos de tipo material/instrumental como gozar de una buena paga, ser capacitado o que esté garantizada la estabilidad laboral y económica. En relación con variables organizacionales e individuales, se observó que en las organizaciones cuyas actividades y roles están vinculados a aspectos humanitarios (e.g. escuelas) se perciben -en mayor medida- cualidades de tipo afectivo/sociales (e.g. valores, compromiso y confianza). Asimismo, este tipo de atributos tienden a ser percibidos por los empleados de mayor edad y con personal a cargo.

Además, en la investigación más reciente (Lupano Perugini \& Castro Solano, 2017b), se observó que los altos niveles de virtudes organizacionales se asociaron con características positivas altruistas como el respeto por la diversidad y la importancia de los valores, en tanto que los niveles bajos de virtudes lo hicieron con características como estabilidad, comodidad y remuneración, lo que indicaría que ante una menor presencia de cualidades morales percibidas en las organizaciones, tendería a privilegiarse aspectos netamente materiales/instrumentales. Resultados que se encuentran en consonancia con los obtenidos en el trabajo anterior.

Sobre la base de los antecedentes teóricos y empíricos expuestos se plantean los siguientes objetivos:

- Analizar diferencias en las virtudes organizacionales percibidas (Apoyo y respeto, Significado e inspiración, Perdón) según variables individuales (género, edad, personal a cargo, tiempo en la organización).

- Analizar diferencias en las virtudes organizacionales percibidas (Apoyo y respeto, Significado e inspiración, Perdón) según variables organizacionales:

- Rubro de la organización (estereotípico, no estereotípico, indefinido)

- Tamaño de la organización (pequeña, mediana, grande)

- Tipo de la organización (pública, privada)

- Analizar si existe relación entre las virtudes organizacionales percibidas (Apoyo y respeto, Significado e inspiración, Perdón) y las siguientes variables de resultado:

- Nivel de performance del empleado y de la organización

- Nivel de satisfacción laboral del empleado 


\section{Método}

\section{Participantes}

Se empleó una muestra voluntaria de 569 empleados (288 hombres, 50.6\%; 281 mujeres, 49.4\%), que tenían una edad promedio de 36.85 años $(D E=11.7)$. La mayoría residía en Ciudad de Buenos Aires -CABA- y provincia de Buenos Aires $(96.8 \%, n=545)$, el 3.9\% ( $n=22)$ en provincias del interior de Argentina y sólo el $0.4 \%(n=2)$ vivía transitoriamente en el exterior. En cuanto al estado civil, el 39.9\% $(n=227)$ estaba casado o en convivencia, el 33.2\% $(n=189)$ era soltero/a, el 17.9\% $(n=102)$ estaba en pareja, el 8.6\% $(n=49)$ estaba separado y sólo el $0.4 \%(n=2)$ no aportó datos. En relación con la clase social percibida, la mayoría se autodescribió como de clase media $(67.5 \%, n=384)$, en tanto que el $17.6 \%$ lo hizo en la clase baja y media-baja $(n=100)$ y el $14.9 \%$ en la clase alta y media-alta $(n=85)$. En cuanto al nivel de estudios el $45.9 \%(n=261)$ poseía estudios primarios y secundarios en tanto que el $54.1 \%(n=308)$ tenía estudios universitarios o de posgrado. Del total de participantes sólo el 33.9\% $(n=193)$ tenían personal a cargo, en tanto que el resto ocupaba una posición de subordinado $(66.1 \%, n=376)$.

En relación con las variables organizacionales, el $81.9 \%(n=466)$ de los empleados pertenecían a empresas privadas, en tanto que el 18.1\% $(n=103)$ lo era de empresas públicas. En relación con el tamaño de la empresa, el 42.4\% ( $n$ $=241)$ pertenecía a grandes empresas, el $33.9 \%(n=193)$ a medianas y el $23.7 \%$ a pequeñas $(n=135)$. Por último, a los fines del presente estudio, se realizó una recategorización de las organizaciones de acuerdo con su rubro. Siguiendo una propuesta de Cameron y colaboradores llevada adelante en dos investigaciones (Cameron et al., 2011), se consideró organizaciones prototípicas/estereotípicas a aquellas cuyas prácticas se relacionan con aspectos positivos a nivel humano, como organizaciones vinculadas a la salud o a la educación. En cambio, serían no prototípicas/no estereotípicas aquellas que destacan principalmente valores materiales como en el caso de las entidades financieras. Por último, se codificó como indefinidas aquellas que no pueden encuadrarse en ninguna de las anteriores, como por ejemplo empresas dedicadas al entretenimiento y ocio (productoras de TV, empresas gastronómicas), y otras por el estilo. El porcentaje de organizaciones prototípicas/estereotípicas fue de 20,7\% $(n=118)$, el de no prototípicas/no estereotípicas $63.4 \%(n=361)$, y el indefinidas $15.8 \%(n=90)$.

\section{Materiales}

Inventario de Virtudes Organizacionales -IVO- (Lupano Perugini \& Castro Solano, 2017a): se trata de una adaptación argentina (Lupano Perugini \& Castro Solano, 2017a) de la prueba original -Positive Practices Survey- (Cameron et 
al., 2011). Este instrumento está destinado a evaluar prácticas virtuosas a nivel organizacional. La versión empleada en este estudio consta de 13 ítems con formato de respuesta Likert de cinco puntos, con recorrido de 1 (completamente en desacuerdo) a 5 (totalmente de acuerdo). Las dimensiones de la prueba son: Apoyo y respeto (las personas se tratan con respeto y muestran conducta de apoyo y colaboración para con los demás), Significado e inspiración (se enfatiza el valor y significado del trabajo, las personas se sienten reconfortadas y renovadas por la tarea que realizan y se inspiran mutuamente en sus trabajos) y Perdón (se evita culpar y se perdonan errores). En la muestra empleada en este estudio, se han obtenido buenos índices de consistencia interna a través del coeficiente alfa de Cronbach (Apoyo y respeto $=.89$; Significado e inspiración $=.87$; Perdón $=.75$ ).

Además, sobre la base de la bibliografía consultada (e.g., Cameron et al., 2004; Cameron et al., 2011), se diseñaron una serie de protocolos para la evaluación de las variables involucradas. Dichos protocolos fueron testeados previamente en un estudio piloto realizado con un grupo reducido de empleados $(n=15)$. Se modificaron algunas expresiones lingüísticas sugeridas por los participantes del estudio piloto dando lugar a las versiones definitivas utilizadas. A continuación se describen los protocolos diseñados:

Encuesta Sociodemográfica: recaba datos personales de los participantes (sexo, edad, lugar de residencia, estado civil, nivel socioeconómico, nivel de estudios, ocupación, nivel socio-económico).

Encuesta Organizacional: consulta a los participantes sobre datos de la organización en la que trabajan y el puesto que ocupan (tamaño, tipo, rubro, área en la que trabaja, puesto y personal a cargo). Además, incluye dos ítems en los cuales se solicita que listen tres características positivas y tres negativas que asocian a la organización (i.e. "Enumere tres características que Ud. considere positivas de la organización en la que trabaja"). Dichas características fueron analizadas en un estudio previo (Lupano Perugini \& Castro Solano, 2016).

Encuesta de Satisfacción Laboral: se diseñaron seis ítems con opción Likert de respuesta que van de 1 (totalmente insatisfecho) a 7 (totalmente satisfecho) que intentan evaluar cuan satisfecho se autopercibe la persona en cuanto a su trabajo en general y diferentes aspectos del mismo (i.e., sueldo, jefes, compañeros, lugar, carrera). Un ejemplo de ítem es “¿Cuán satisfecho estoy con el sueldo que recibo?”. Para la elección de las áreas a evaluar (e.g. sueldo, jefes, compañeros), se tomaron en cuenta aspectos analizados en otros instrumentos previos (e.g., Balzer et al., 1997). Se calculó alfa de Cronbach para la escala total obteniéndose un valor aceptable de .76

Encuesta sobre Desempeño-performance Organizacional e Individual: Frente a la imposibilidad de obtener indicadores objetivos sobre el rendimiento 
organizacional se decidió inferirlo a partir de la percepción de los empleados. Se tomaron como indicadores de desempeño organizacional algunos utilizados por Cameron et al., (2004) en sus investigaciones sobre virtudes y performance. Los mismos aluden a niveles de eficiencia, innovación, crecimiento, calidad, retención de empleados y clientes, satisfacción, adaptación. Por ejemplo, para el diseño de la primera sección de la encuesta -orientada a la evaluación del desempeño organizacional- se construyeron diez ítems con opción Likert de respuesta -1 (Poco) a 6 (Mucho) -, considerando los indicadores de eficiencia propuestos por Cameron et al., (2004) (e.g. cumplimento de objetivos, atención a las demandas del cliente, utilización óptima de recursos, etc). Un ejemplo de ítem es "En qué medida cree que la organización cumplió eficientemente -haciendo un buen uso de los recursos- con los objetivos propuestos". El alfa obtenido para este apartado fue de .89. Siguiendo el mismo criterio, se diseñó otra sección que solicitaba al participante que califique su desempeño como empleado. Se diseñaron seis ítems con la misma opción de respuesta que el apartado anterior $(1=$ Poco y $6=$ Mucho). Un ejemplo de ítem es: "En qué medida cree que los resultados obtenidos fueron de calidad". El alfa obtenido para esta sección fue de .84 .

\section{Procedimiento}

Los datos fueron recolectados por alumnos que se encontraban realizando una práctica de investigación en una universidad privada de la ciudad de Buenos Aires. Los participantes fueron voluntarios y no recibieron retribución alguna por su colaboración. Además, el cuadernillo que contenía las encuestas presentaba en su portada una introducción en la que se solicitaba el consentimiento del participante, se aseguraba el anonimato de los datos y su uso exclusivo para investigación. La recolección y carga de datos fue supervisado por un docente investigador.

\section{Tipo de estudio y diseño}

Estudio correlacional - de diferencias de grupos. Diseño transversal.

\section{Análisis de datos}

En primer lugar, se calcularon estadísticos descriptivos para las variables analizadas, incluyendo los valores de asimetría y curtosis a fin de testear la normalidad de los datos. Luego se efectuaron análisis de diferencias de medias mediante el uso de prueba t y ANOVA de un factor. Por último, se realizaron correlaciones de Pearson para estimar asociaciones entre las variables involucradas. El programa empleado para la carga y análisis de los datos fue SPPS 18.0 . 


\section{Resultados}

En primer lugar, se calcularon estadísticos descriptivos para las tres dimensiones de la escala de virtudes organizacionales y para las variables satisfacción laboral, performance organizacional e individual. Como puede observarse en la tabla 1, todas las variables obtuvieron valores en torno al término medio. Además, los valores de asimetría y curtosis obtenidos señalan normalidad univariada de las variables analizadas.

Tabla 1.

Estadísticos descriptivos de las variables analizadas

\begin{tabular}{lccc}
\hline Variables & $M(D E)$ & Asimetría & Curtosis \\
\hline Apoyo y respeto & $3.54(0.72)$ & -0.64 & -0.83 \\
Significado e inspiración & $3.22(0.88)$ & -0.32 & -0.15 \\
Perdón & $3.19(0.84)$ & -0.18 & -0.33 \\
Satisfacción laboral & $4.89(1.04)$ & -0.58 & -0.47 \\
Performance organizacional & $4.07(0.92)$ & -0.47 & -0.04 \\
Performance individual & $4.61(0.83)$ & -0.61 & -0.47
\end{tabular}

A continuación, con la finalidad de responder al objetivo 1 que intentaba analizar diferencias en las virtudes organizacionales percibidas según variables individuales (género, edad, personal a cargo y tiempo en la organización), se calcularon diversos análisis de pruebas t. Se recategorizó la variable edad en menores de 40 años $(n=383)$ y mayores de 40 años $(n=186)$. Asimismo, a la variable tiempo en la organización se la recategorizó en permanencia menor a 5 años $(n=306)$ y mayor a 5 años $(n=263)$ (ver tablas 2 y 3$)$. 
Tabla 2.

Diferencia de medias según género y edad

\begin{tabular}{lcccccc}
\hline Dimensiones & $\begin{array}{c}\text { Mujeres } \\
(\mathrm{n}=281) \\
M(D E)\end{array}$ & $\begin{array}{c}\text { Varones } \\
(\mathrm{n}=288) \\
M(D E)\end{array}$ & $\boldsymbol{t}$ & $\begin{array}{c}<40 \\
(\mathrm{n}=383) \\
M(D E)\end{array}$ & $\begin{array}{c}>40 \\
(\mathrm{n}=186)\end{array}$ & $\boldsymbol{M}(\boldsymbol{D E})$ \\
\hline Apoyo y respeto & $3.53(0.72)$ & $3.54(0.71)$ & $0.18 \mathrm{~ns}$ & $3.50(0.71)$ & $3.59(0.72)$ & $1.34 \mathrm{~ns}$ \\
$\begin{array}{l}\text { Significado e } \\
\text { inspiración }\end{array}$ & $3.20(0.87)$ & $3.24(0.88)$ & $0.46 \mathrm{~ns}$ & $3.13(0.89)$ & $3.40(0.83)$ & $3.51^{\star \star}$ \\
Perdón & $3.16(0.82)$ & $3.17(0.89)$ & $0.19 \mathrm{~ns}$ & $3.14(0.85)$ & $3.19(0.79)$ & $0.54 \mathrm{~ns}$ \\
\hline${ }^{* \star} \mathrm{p}<.001$ & & & & & &
\end{tabular}

Tabla 3.

Diferencia de medias según personal a cargo y tiempo en la organización

\begin{tabular}{lcccccc}
\hline Dimensiones & $\begin{array}{c}\text { Con perso- } \\
\text { nal } \\
(\mathrm{n}=193) \\
M(D E)\end{array}$ & $\begin{array}{c}\text { Sin personal } \\
(\mathrm{n}=376) \\
M(D E)\end{array}$ & $\boldsymbol{t}$ & $\begin{array}{c}<5 \text { años } \\
(\mathrm{n}=306) \\
M(D E)\end{array}$ & $\begin{array}{c}>5 \text { años } \\
(\mathrm{n}=263) \\
M(D E)\end{array}$ & $t$ \\
\hline $\begin{array}{l}\text { Apoyo y } \\
\text { respeto }\end{array}$ & $3.60(0.68)$ & $3.50(0.73)$ & $1.62 \mathrm{~ns}$ & $\begin{array}{c}3.56 \\
(0.69)\end{array}$ & $\begin{array}{c}3.50 \\
(0.73)\end{array}$ & $0.86 \mathrm{~ns}$ \\
$\begin{array}{l}\text { Significado e } \\
\text { inspiración }\end{array}$ & $3.37(0.82)$ & $3.15(0.90)$ & $2.98^{\star *}$ & $\begin{array}{c}3.18 \\
(0.89)\end{array}$ & $\begin{array}{c}3.27 \\
(0.88)\end{array}$ & $1.25 \mathrm{~ns}$ \\
Perdón & $3.17(0.83)$ & $3.16(0.83)$ & $0.11 \mathrm{~ns}$ & $\begin{array}{c}3.22 \\
(0.84)\end{array}$ & $\begin{array}{c}3.09 \\
(0.82)\end{array}$ & $1.78 \mathrm{~ns}$ \\
\hline
\end{tabular}

${ }^{\star \star} p<.001$

Los resultados de las tablas 2 y 3 muestran que no se han encontrado diferencias individuales en las virtudes analizadas de acuerdo al género del participante. En cambio, en cuanto a la edad, se han hallado diferencias estadísticamente significativas en la dimensión Significado e Inspiración a favor de los mayores de 40 años, no así en las otras dos virtudes analizadas. Con relación a si contaban o no con personal a cargo, también se encontraron diferencias significativas - a favor de los que tenían subordinados- en la dimensión Significado e Inspiración. Por último, no se hallaron diferencias tomando en cuenta la antigüedad en la organización.

Con la finalidad de responder al objetivo 2 que intentaba analizar diferencias en las virtudes organizacionales percibidas según variables organizacionales, se calculó prueba $t$ y ANOVA de un factor.

Se encontraron diferencias estadísticamente significativas de acuerdo al tipo de empresa sólo en la dimensión Apoyo y respeto a favor de las organizaciones privadas $\left(t=2.17, p=.03, M_{\text {privadas Apoyo }=} 3.57, M_{\text {públicas Apoyo }}=3.39 ; t=.80, p=.43\right.$, 
$M_{\text {privadas Significado }}=3.21, M_{\text {públicas Significado }}=3.29 ; t=.44, p=.66, M_{\text {privadas Perdón }}=3.17$, $M_{\text {públicas Perdón }}=3.12$ ). Por otro lado, mediante análisis de ANOVA de un factor se halló diferencias significativas en la dimensión Apoyo y respeto según tamaño de la empresa $[F(2,566)=4.04, p=.00]$. En cambio, no se han encontrado diferencias en la dimensión Significado e inspiración $[F(2,566)=1.32, p=.27]$, y tampoco en la virtud Perdón $[F(2,566)=1.24, p=.29]$. Mediante contrastes post hoc Tukey-b se determinó que en las empresas de tamaño grande se perciben menores niveles de apoyo y respeto que en las pequeñas y medianas (ver tabla 4). En relación con el rubro de la empresa, se obtuvieron diferencias significativas en las dimensiones Apoyo y respeto $[F(2,566)=5.41, p=.00]$, y Significado e inspiración $[F(2,566)=9.49, p=.00]$. A través de contrastes post hoc Tukey-b se observó que en las empresas de rubro no estereotípico se perciben menores niveles de apoyo e inspiración (ver tabla 4).

Tabla 4.

Estadísticos Descriptivos. ANOVA de un factor.

\begin{tabular}{llcccccccc}
\hline & & \multicolumn{2}{c}{$\begin{array}{c}\text { Apoyo y } \\
\text { respeto }\end{array}$} & $\begin{array}{c}\text { Significado e } \\
\text { inspiración }\end{array}$ & Perdón & Tukey-b \\
& & M & DE & M & DE & M & DE & \\
\multirow{5}{*}{ Tamaño } & Pequeña (a) & 3.60 & 0.77 & 3.27 & 0.94 & 3.25 & 0.84 & \\
& Mediana (b) & 3.61 & 0.66 & 3.28 & 0.86 & 3.16 & 0.82 & Grupos \\
& Grande (c) & 3.44 & 0.72 & 3.15 & 0.86 & 3.11 & 0.84 & c y a-b \\
\multirow{3}{*}{ Rubro } & Estereotípico (a) & 3.68 & 0.74 & 3.49 & 0.86 & 3.21 & 0.79 & \\
& No-estereotípico (b) & 3.46 & 0.69 & 3.10 & 0.88 & 3.10 & 0.84 & Grupos \\
& Indefinido (c) & 3.65 & 0.74 & 3.34 & 0.85 & 3.33 & 0.86 & b y a-c \\
\hline
\end{tabular}

Por último, a fin de responder al tercer objetivo que buscaba analizar si existe relación entre las virtudes organizacionales percibidas y los niveles de performance del empleado/ organización y de satisfacción laboral del empleado, se calcularon coeficientes de correlación de Pearson. Como se puede observar en la tabla 5, todas las correlaciones obtenidas son significativas. Además, la mayoría ha obtenido un tamaño del efecto de moderado $(r \mathrm{~s} \geq .30)$ a grande $(r \mathrm{~s}$ $\geq .50$ ) (Cohen, 1992). Las correlaciones más altas se han obtenido entre la virtud Significado e inspiración y las tres variables de resultado. 
Tabla 5.

Correlaciones entre virtudes organizacionales y variables de resultado.

\begin{tabular}{lccc}
\hline & $\begin{array}{c}\text { Performance } \\
\text { organizacional }\end{array}$ & $\begin{array}{c}\text { Performance } \\
\text { del empleado }\end{array}$ & $\begin{array}{c}\text { Satisfacción laboral } \\
\text { del empleado }\end{array}$ \\
\hline Apoyo y respeto & $.44^{\star *}$ & $.28^{\star \star}$ & $.51^{\star \star}$ \\
Significado e inspiración & $.44^{\star *}$ & $.46^{\star \star}$ & $.55^{\star *}$ \\
Perdón & $.28^{* *}$ & $.18^{\star *}$ & $.30^{\star *}$ \\
\hline
\end{tabular}

** $p<.001$

\section{Comentarios}

El presente trabajo proponía una serie de objetivos tendientes a buscar diferencias en las virtudes organizacionales de acuerdo a variables individuales y organizacionales y, además, determinar la relación con medidas de resultado. Sobre la base de lo obtenido, se concluye que la única dimensión que ha introducido diferencias en cuanto a variables individuales es la de Significado e inspiración. Puntualmente, se halló que las personas de mayor edad y los que ocupan una posición de liderazgo tienden a percibir que en la organización en la que desempeñan sus funciones se la da importancia al trabajo que realizan y se los motiva e inspira a seguir realizándolo. Estos resultados son congruentes con lo hallado en un trabajo previo (Lupano Perugini \& Castro Solano, 2016) en el que se observó que los mayores de 45 años y con personal a cargo destacaron una serie de atributos positivos de las organizaciones en las que trabajan -como el prestigio y el compromiso-, dando cuenta del valor que se le otorga al trabajo y los resultados obtenidos. Por otro lado, considerando variables organizacionales, se halló que en las empresas de tamaño grande se perciben menores niveles de apoyo. Esto podría indicar que las prácticas relacionadas con la preocupación, confianza y amabilidad hacia los demás se ven obstaculizadas en aquellas organizaciones en las que se dificulta el contacto personalizado debido al gran tamaño de las mismas. Además, estas empresas pueden estar teñidas de excesiva burocracia -atributo que se identificó como frecuente en las organizaciones grandes y públicas de Argentina (Lupano Perugini \& Castro Solano, 2016)-. Asimismo, en las empresas de rubro no estereotípico se observó que se perciben menores niveles de apoyo e inspiración. Esto resulta congruente con una investigación previa (Lupano Perugini \& Castro Solano, 2016) en la que se halló que los empleados de empresas de rubro no estereotípico (e.g. bancos y otras entidades financieras) percibían atributos materiales/instrumentales (e.g. buena remuneración) en detrimento 
de factores personales y/o afectivos. Por último, las tres virtudes analizadas correlacionaron con medidas de satisfacción y performance, principalmente la virtud Significado e inspiración. Este resultado señalaría que los empleados que rinden mejor, que creen que su organización ha obtenido buenos resultados y, a su vez, se sienten más satisfechos, son los que perciben que en dicha organización se le da relevancia al trabajo que realizan y esto los hace sentir renovados e inspirados para seguir realizando lo que hacen. Estos datos se relacionan con las investigaciones de Cameron et al. (2011) en las que comprobaron el efecto positivo que presentan las prácticas virtuosas a nivel organizacional sobre los niveles de performance en diferente tipo de organizaciones.

Sería interesante que los resultados obtenidos fueran tenidos en cuenta en el área aplicada. Las áreas de Personal o Recursos Humanos de las organizaciones podrían implementar estrategias que fomenten la importancia del trabajo en los más jóvenes ya que los niveles más altos de la virtud Significado se obtuvieron en los sujetos mayores y con personal a cargo. Deberían propiciarse actividades, sobre todo de parte de quienes ocupan una posición de liderazgo, en las que se trate de reflexionar acerca de la importancia de la labor que se realiza, generando así un clima más motivador. Asimismo, sería importante incrementar los niveles de apoyo y respeto en aquellas organizaciones de gran tamaño y/o que pertenecen a rubros no relacionados con aspectos sociales.

Se puede destacar como fortaleza del estudio realizado el impulso al desarrollo a nivel latinoamericano de estudios organizacionales basados en una orientación positiva, situación que ha venido creciendo en los últimos años. Además, los resultados obtenidos contemplan la idiosincrasia y necesidades locales y alientan a no aplicar conclusiones obtenidas en contextos culturalmente diferentes, sin una previa adaptación. En cuanto a limitaciones posibles, se puede mencionar el empleo de medidas subjetivas para estimar el desempeño individual y organizacional. Asimismo, la muestra empleada podría balancearse en cuanto al porcentaje de empresas públicas y privadas incluidas.

Por último, futuros estudios deberían continuar con el análisis de estos aspectos a través de diseños multivariados en los que se incluyan otras variables estudiadas en estudios organizacionales (como puede ser el work engagement $\mathrm{o}$ vinculación psicológica con el trabajo, capital psicológico, emociones positivas, conducta prosocial, etc.) que pueden estar relacionadas con la obtención de resultados eficaces tanto a nivel individual como organizacional. Además, este tipo de diseños permitirá analizar el peso individual que cada virtud puede tener sobre dichas variables de resultado, aspecto que según Cameron y otros (Cameron et al., 2011; Cameron \& Winn, 2012) resulta difícil de predecir. 


\section{Referencias}

Balzer, W. K., Kihm, J. A., Smith, P. C., Irwin, J. L., Bachiochi, P. D., Robie, C.,... Parra, L. F. (1997). User's manual for the Job Descriptive Index (JDI; 1997 Revision) and the Job in General (JIG) Scales. Bowling Green, OH: Bowling Green State University.

Bright, D. S., Cameron, K. S., \& Caza, A. (2006). The Amplifying and Buffering Effects of Virtuousness in Downsized Organizations. Journal of Business Ethics, 64, 249-269. https://doi.org/10.1007/s10551-005-5904-4

Cameron, K. S., Brigth, D., \& Caza, A. (2004). Exploring the relationship between organizational virtuosness and performance. American Behavioral Scientist, 47(6), 1-24. https://doi.org/10.1177/0002764203260209

Cameron, K. S., Mora, C., Leutscher, T., \& Calarco, M. (2011). Effects of positive practices on organizational effectiveness. The Journal of applied Behavioral Science, 47, 266-308. https://doi.org/10.1177/0021886310395514

Cameron, K. S. \& Spreitzer, G. M. (2012). What is positive about Positive Organizational Scholarship? En K. S. Cameron \& G. M. Spreitzer (Eds.), The Oxford Handbook of Positive Organizational Scholarship (pp. 1-14). New York: Oxford University Press.

Cameron, K. S. \& Winn, B. (2012). Virtuousness in Organizations. In: K. S. Cameron \& G. M. Spreitzer (Eds.), The Oxford Handbook of Positive Organizational Scholarship (pp. 231-243). New York: Oxford University Press.

Cohen, J. (1992). A power primer. Psychological Bulletin, 112(1), 155-159. https:// doi.org/10.1037/0033-2909.112.1.155

Dutton, J. E. \& Glynn, M. (2007). Positive Organizational Scholarship. En C. Cooper \& J. Barling (Eds.), Handbook of organizational behavior. Thousand Oaks, CA: Sage.

Fineman, S. (2006). On being positive: Concerns and counterpoints. Academy of Management Review, 31, 270-291. https://doi.org/10.5465/ AMR.2006.20208680

Judge, T. A., Thorensen, C. J., Bono, J. E., \& Patton, G. K. (2001). The job satisfaction-job performance relationship: A qualitative and quantitative 
review. Psychological Bulletin, 127, 376-407. https://doi.org/10.1037/00332909.127.3.376

Lupano Perugini, M. L. (2014). Organizaciones Positivas: un marco de referencia para su abordaje en Latinoamérica. Acta Psiquiátrica y Psicológica de América Latina, 60(4), 277-284.

Lupano Perugini \& Castro Solano, A. (2016). Perfiles de organizaciones positivas. Análisis de características percibidas según variables individuales, organizacionales y de resultado. Escritos de Psicología, 9(2), 1-11. https://doi. org/10.5231/psy.writ.2016.1103

Lupano Perugini, M. L. \& Castro Solano, A. (2017a). Influencia de las Virtudes Organizacionales sobre la performance laboral. Un estudio en organizaciones argentinas. Interdisciplinaria, en prensa.

Lupano Perugini, M. L. \& Castro Solano, A. (2017b). Virtudes organizacionales y características positivas asociadas a performance y satisfacción laboral. Acción Psicológica, en prensa.

Park, N. \& Peterson, Ch. (2003). Virtues and organizations. En K. S. Cameron, J. E., Dutton, \& R. E. Quinn (Eds.), Positive organizational scholarship: Foundations of a new discipline (pp. 33-47). San Francisco: Berrett-Koehler.

Peterson, Ch. (2006). Enabling Institutions. En Ch. Peterson (Ed.), A primer in Positive Psychology (pp. 275-304). New York: Oxford University Press.

Peterson, C. \& Seligman, M. E. P. (2004). Character strengths and virtues: A handbook and classification. New York, NY, US: Oxford University Press. REFERENCIA NO CITADA

Quinn, R. (2015). The Positive Organization: Breaking Free from Conventional Cultures, Constraints, and Beliefs. Oakland: CA: Berrett-Koehler. https://doi. org/10.5848/bk.978-1-62656-563-0_3

Salanova, M., Llorens, S., \& Martínez, I.M. (2016). Aportaciones desde la Psicología Organizacional Positiva para desarrollar organizaciones saludables y resilientes. Papeles del Psicólogo, 37(3), 177-184.

Siedel, G. J. (2016). The three pillars Model for Business Decisions. Michigan: Van Rye Publishing, LLC.

Spector, P. E. (1997). Job Satisfaction: Application, Assessment, Causes, and 
Consequences. United Kingdom: Sage Publications Ltd.

Weiss, H. M. \& Brief, A. P. (2001). Affect at work: A historical perspective. In: R. L. Payne \& C. L. Cooper (Eds), Emotions at Work: Theory, Research, and Applications for Management (pp. 133-172). Chichester, UK: Wiley. 